\title{
Case Report \\ Pulmonary Aspergilloma Treated by Limited Thoracoplasty with Simultaneous Cavernostomy and Muscle Transposition Flap
}

\author{
Hitoshi Igai, MD, PhD, Mitsuhiro Kamiyoshihara, MD, PhD, \\ Toshiteru Nagashima, MD, and Yoichi Ohtaki, MD
}

\begin{abstract}
We describe the successful treatment of pulmonary aspergilloma by limited thoracoplasty used simultaneously with single-stage cavernostomy and a muscle transposition flap. An 80-year-old man with dyspnea on effort and hemoptysis consulted our hospital. Chest computed tomography revealed a thick wall cavity containing a fungus ball surrounded by a crescent of air and diseased lung parenchyma, indicating complex pulmonary aspergilloma (CPA). As curative pulmonary resection was considered too invasive for this patient, limited thoracoplasty with simultaneous single-stage cavernostomy and myoplasty was performed as an alternative treatment. During the operation, transposition flap of the latissimus dorsi muscle was created by preserving the feeding artery. Fungus, in the ball that was removed, proved to be aspergilloma by culture and histopathological examination. On postoperative day 15 , the patient was discharged uneventfully, and 4 months after surgery, no relapse of the aspergilloma has been observed.

We consider that limited thoracoplasty with simultaneous single-stage cavernostomy and a muscle transposition flap is effective for treatment of high-risk CPA.
\end{abstract}

Keywords: pulmonary aspergilloma, limited thoracoplasty, single-stage cavernostomy, muscle transposition flap

\section{Introduction}

Lung resection for complex pulmonary aspergilloma (CPA) is considered to be the most effective form of curative therapy. However, surgical resection for patients with CPA is associated with significant morbidity and mortality because of poor pulmonary function, general condition, or bilateral disease. ${ }^{1)}$ Therefore, successful results of single-stage cavernostomy and myoplasty as palliative

Department of General Thoracic Surgery, Maebashi Red Cross Hospital, Maebashi, Gunma, Japan

Received: August 8, 2011; Accepted: November 30, 2011

Corresponding author: Hitoshi Igai, MD, PhD. Department of General Thoracic Surgery, Maebashi Red Cross Hospital, 3-21-36 Asahi-cho, Maebashi, Gunma 371-0014, Japan

Email: hitoshi-iga@hotmail.co.jp

(C)2012 The Editorial Committee of Annals of Thoracic and Cardiovascular Surgery. All rights reserved. therapy for these high-risk patients have been reported by several authors. ${ }^{2-4)}$ In this form of treatment, muscle flap disuse atrophy is sometimes a cause of failure. Here, we report a case of pulmonary aspergilloma that was treated successfully by limited thoracoplasty with simultaneous single-stage cavernostomy and a muscle transposition flap, and review the relevant literature.

\section{Case Report}

An 80-year-old man with dyspnea on effort and hemoptysis consulted our hospital. Initial chest X-ray examination and computed tomography revealed a thick wall cavity containing a fungus ball surrounded by a crescent of air and diseased lung parenchyma (Fig. 1A and 1B). Serum antibody against aspergilloma was positive. Therefore, the patient was diagnosed with CPA clinically, and surgical treatment was planned. Because 


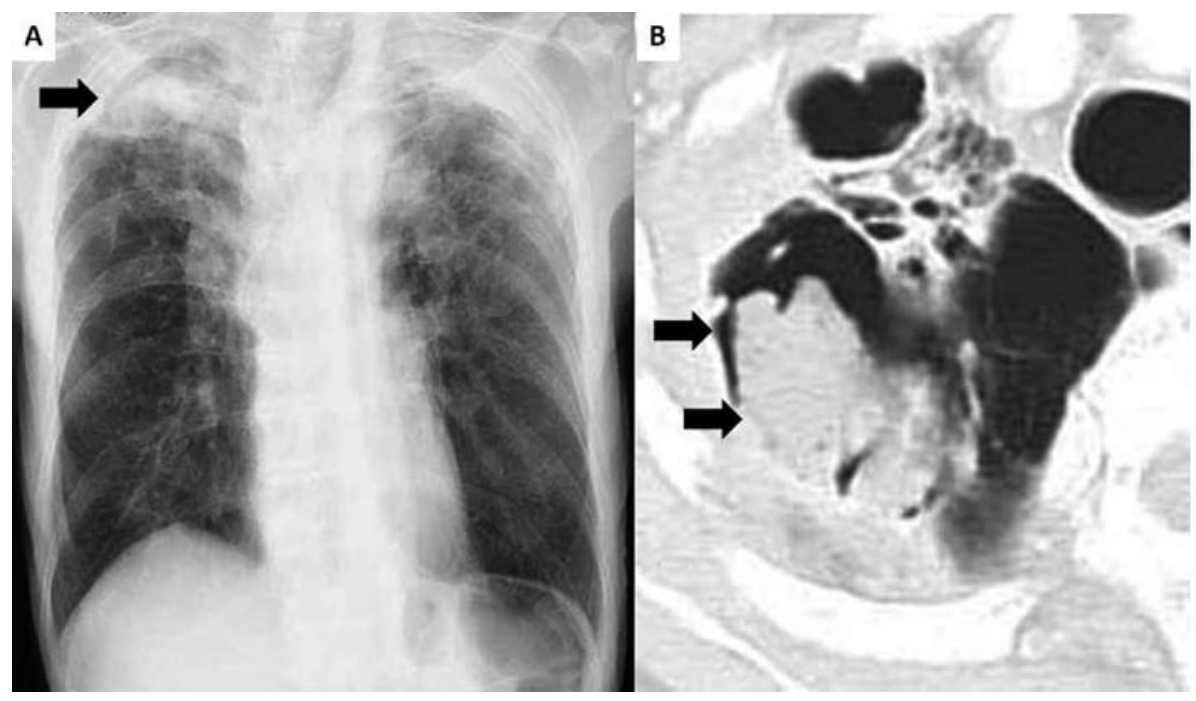

Fig. 1 Chest X-ray (A) and computed tomography (B) revealed a thick wall cavity containing a fungus ball (arrows) surrounded by a crescent of air and diseased lung parenchyma.

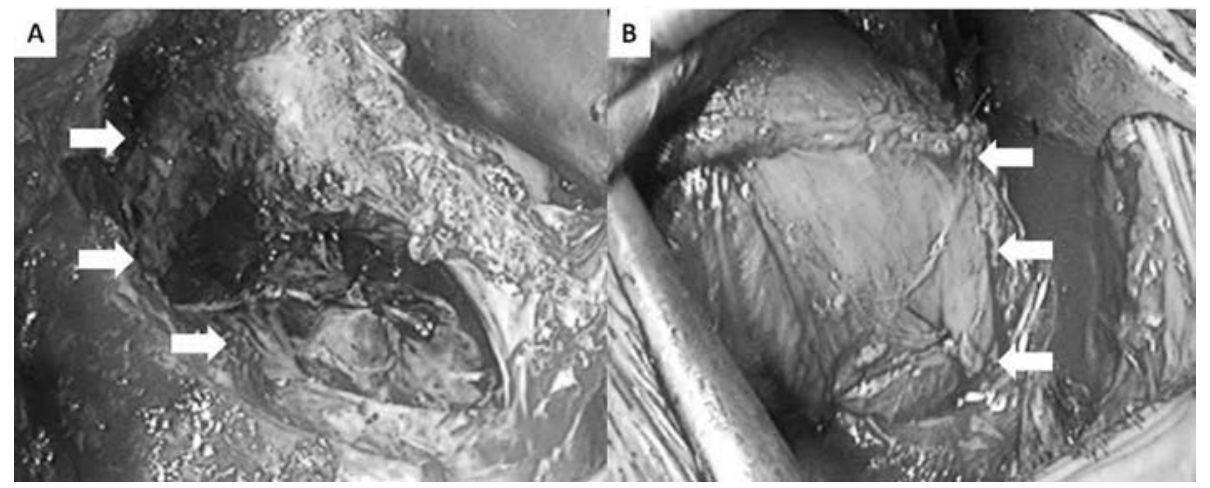

Fig. 2 After the fungus ball had been removed and the cavity irrigated (A), the transposition flap of the latissimus dorsi muscle (arrows) was fixed with 3-0 monofilament suture and occupied the cavity (B).

pulmonary resection was considered too invasive for this patient, limited thoracoplasty with simultaneous singlestage cavernostomy and a transposition flap of the latissimus dorsi muscle was performed as an alternative treatment.

After double-lumen endotracheal intubation, the patient was placed in the lateral decubitus position. A $10-\mathrm{cm}$ lateral skin incision along the $3 \mathrm{rd}$ rib was made, and a thoracotomy in the 3rd rib bed was performed. Subsequently, the 3rd rib was excised. After the incising the hypertrophic parietal pleura, emphysematous lung parenchyma, which adhered to the parietal pleura, was encountered. After dissecting the adhesion toward the back, we approached the cavity containing the fungus ball. The fungus ball was then removed and examined by bacterial and fungal culture, and the histopathological diagnosis made from the specimen was aspergilloma.

After the cavity had been irrigated with physiological saline (Fig. 2A), a sealing test $\left(15 \mathrm{cmH}_{2} \mathrm{O}\right.$ pressure) was performed, and this demonstrated several points of bron- chial openings ventilated with high pressure. After making an additional skin incision, we created a transposition flap of the latissimus dorsi muscle while preserving the feeding artery.

After fibrin glue had been sprayed into the cavity, we fixed the transposition flap of the latissimus dorsi muscle with 3-0 monofilament suture so that the flap could occupy the cavity (Fig. 2B). The total operation time was 150 min, and total blood loss was $20 \mathrm{~g}$. Postoperative X-ray examination confirmed that the cavity was occupied by the transposition flap of the muscle (Fig. 3).

On postoperative day 15 , the patient was discharged uneventfully, and no relapse of the pulmonary aspergilloma has been observed after 4 months of postoperative follow-up.

\section{Discussion}

In the present case of CPA, we successfully performed a limited thoracoplasty with simultaneous single-stage 


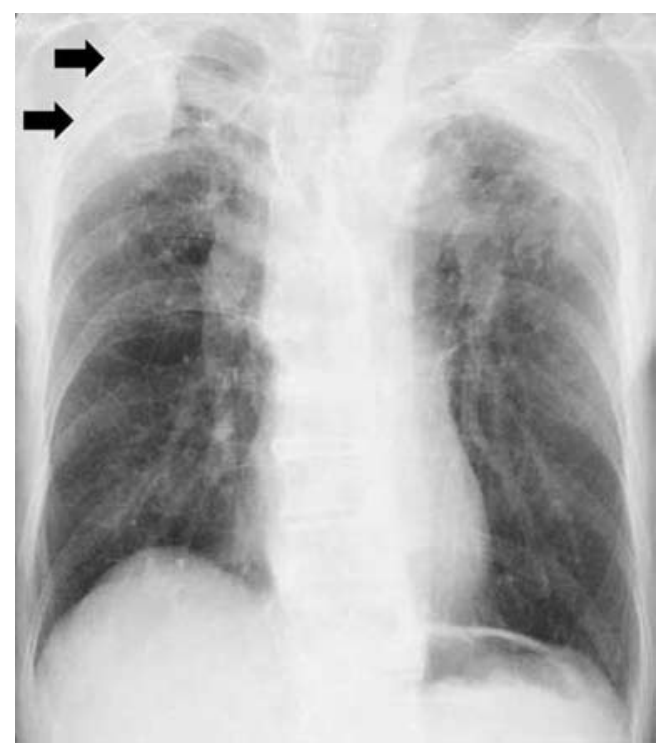

Fig. 3 Postoperative X-ray examination confirmed that the cavity was occupied by the transposition flap of the latissimus dorsi muscle and limited thoracoplasty.

cavernostomy and placement of a transposition flap of the latissimus dorsi muscle.

Although lung resection is the optimal curative treatment for pulmonary aspergilloma, several authors have reported that single-stage cavernostomy and myoplasty is an effective alternative therapy for this condition, since Daly et al. reported its use for the first time..$^{2-4)}$

One reported cause of failure in this treatment is muscle flap disuse atrophy. ${ }^{3)}$

In our patient, the following were considered important for obtaining a successful result:

1. Preservation of the feeding artery of the muscle flap

2. Reduction of the cavity volume by resection of a rib, so-called "limited thoracoplasty"

It has been reported that disuse muscle atrophy frequently occurs in the first 5-6 weeks after transposition, and that this appears to be responsible for partial cavity recurrence. ${ }^{3)}$ Fortunately, the radiograph did not show atrophy of the flap or an increase in cavity volume. Grima et al. reported acceptable results for CPA by limited thoracoplasty with single-stage cavernostomy and myoplasty, as in the present case. ${ }^{3)}$ Therefore, we consider that these techniques are key factors for successful single-stage cavernostomy and myoplasty.

Several reports have described the effectiveness of cavernostomy, ${ }^{5)}$ intracavitary amphotericin- $\mathrm{B},{ }^{6}$ ) or bronchial artery occlusion ${ }^{7}$ as palliative therapies for highrisk patients with CPA. However, it is crucial to ensure that all bronchial openings are closed completely to prevent relapse of aspergilloma in the long term. Therefore, if the condition of the patient allows it, we also include placement of a transposition flap in the treatment, since the flap can close any bronchial openings.

\section{References}

1) Massard G, Roeslin N, Whilm JM, et al. Pleuro-pulmonary aspergilloma: clinical spectrum and results of surgical treatment. Ann Thorac Surg 1992; 54: 1159-64

2) Daly RC, Pairolero PC, Piehler JM, et al. Pulmonary aspergilloma. Results of surgical treatment. J Thorac Cardiovasc surg 1986; 92: 981-8.

3) Grima R, Krassas A, Bagan P, et al. Treatment of complicated pulmonary aspergillomas with cavernostomy and muscle flap: interest of concomitant limited thoracoplasty. Eur J Cardiothorac Surg 2009; 36: 910-3.

4) Gebitekin C, Sami Bayram A, Akin S. Complex pulmonary aspergilloma treated with single-stage cavernostomy and myoplasty. Eur J Cardiothorac Surg 2005; 27: 737-40.

5) Babatasi G, Massetti M, Chapelier A, et al. Surgical treatment of pulmonary aspergilloma: current outcome. J Thorac Cardiovasc Surg 2000; 119: 906-12.

6) Lee KS, Kim HT, Kim H, et al. Treatment of hemoptysis in patients with cavitary aspergilloma. Am J Roentgenol 1993; 161: 727-31.

7) MacErlan DP, Gray BJ, FitzGerald MX. Bronchial artery embolization in the control of massive haemoptysis. Br J Radiol 1979; 52: 558-61. 